\title{
A Predictor-Corrector Method for Solving Equilibrium Problems
}

\author{
Zong-Ke Bao, ${ }^{1,2}$ Ming Huang, ${ }^{2,3}$ and Xi-Qiang Xia ${ }^{2}$ \\ ${ }^{1}$ School of Accounting, Zhejiang University of Finance and Economics, Hangzhou 310018, China \\ ${ }^{2}$ Dalian University of Technology, Dalian 116024, China \\ ${ }^{3}$ Dongbei University of Finance and Economics, Dalian 116025, China
}

Correspondence should be addressed to Ming Huang; huangming0224@163.com

Received 28 April 2014; Accepted 30 June 2014; Published 15 October 2014

Academic Editor: Adrian Petrusel

Copyright (C) 2014 Zong-Ke Bao et al. This is an open access article distributed under the Creative Commons Attribution License, which permits unrestricted use, distribution, and reproduction in any medium, provided the original work is properly cited.

\begin{abstract}
We suggest and analyze a predictor-corrector method for solving nonsmooth convex equilibrium problems based on the auxiliary problem principle. In the main algorithm each stage of computation requires two proximal steps. One step serves to predict the next point; the other helps to correct the new prediction. At the same time, we present convergence analysis under perfect foresight and imperfect one. In particular, we introduce a stopping criterion which gives rise to $\Delta$-stationary points. Moreover, we apply this algorithm for solving the particular case: variational inequalities.
\end{abstract}

\section{Introduction}

Equilibrium problems theory provides us with a unified, natural, innovative, and general framework to study a wide class of problems arising in finance, economics, network analysis, transportation, elasticity, and optimization. This theory has witnessed an explosive growth in theoretical advances and applications across all disciplines of pure and applied sciences. As a result of this interaction, we have a variety of techniques to study existence results for equilibrium problems; see [1-4]. Equilibrium problems include variational inequalities as special cases. In recent years, several numerical techniques [5-12] including projection, resolvent, and auxiliary principle have been developed and analyzed for solving equilibrium problems.

Let $C$ be a nonempty closed convex subset of $R^{n}$, and let $f: C \times C \rightarrow R$ be a continuous function satisfying $f(x, x)=$ 0 for all $x \in C, f(x, \cdot)$ is convex on $C$ for all $x \in C$, and $f(\cdot, y)$ is lower semicontinuous (l.s.c.) on $C$ for all $y \in C$. The equilibrium problems (for short EP) proposed by BlumOettli [1] are as follows:

finding $x^{*} \in C$ such that $f\left(x^{*}, y\right) \geq 0, \quad \forall y \in C$.

Recently, much attention has been given to reformulate the equilibrium problem as an optimization problem. This problem is very general in the sense that it includes, as particular cases, the optimization problem, the variational inequality problem, the Nash equilibrium problem in noncooperative games, the fixed-point problem, the nonlinear complementarity problem, and the vector optimization problem (see, e.g., $[1,13]$ and the references quoted therein). Multiobjective optimization problems can also be obtained by (EP), as shown by Iusem and Sosa [13]. The above particular cases are useful models of many practical problems arising in game theory, physics, economics, and so forth. The interest of this problem is that it unifies all these particular problems in a convenient way. For example, the work of Brezis et al. extended results concerning variational inequalities, corresponding to the case where $f(x, y)=\langle A x, y-x\rangle$ and $A$ is a monotone operator (see [14], pages 296-297). Moreover, many methods devoted to solving one of these problems can be extended, with suitable modifications, to solve the general equilibrium problem. In this paper we suppose that there exists at least one solution to problem (EP). In particular, it is true when $C$ is compact. Other existence results for this problem can be found, for instance, in $[1,15]$.

In this paper, one uses usually the auxiliary principle technique. This technique deals with finding a suitable auxiliary problem and proving that the solution of the auxiliary problem is the solution of the original problem by using the fixed-point approach. Glowinski et al. [6] used this technique to study the existence of a solution of mixed variational inequalities. Noor [8] has used this technique to suggest and analyze a number of iterative methods for solving 
various classes of variational inequalities. It has been shown that a substantial number of numerical methods can be obtained as special cases from this technique. In this paper, we use again the auxiliary principle technique to suggest and analyze some predictor-corrector methods for solving equilibrium problems. In this respect, our results represent an improvement of previously known results. Noor [16] and Noor et al. [17] have introduced inertial proximal methods for variational inequalities using the auxiliary principle technique and proved that the convergence criteria of inertial proximal methods require only pseudomonotonicity. Inertial proximal methods include proximal methods as a special case. For recent development and applications of the proximal methods, see $[5,11,18]$. Our results can be considered as novel and important applications of the auxiliary principle technique. This paper is an extension over the related work of $[19,20]$; the main contributions can be summarized as follows. First of all, we extend the coefficient of approximate function from $\mu \in(0,1]$ to $\mu \in R \backslash\{0\}$, which is a better conclusion. Secondly, approximate function does not need to satisfy the conditions $(C 1)-(C 3)$ in [20]; that is to say, our condition is weaker than therein. Moreover, we present a new algorithm, predictor-corrector methods for solving (EP), and give a stopping criterion. In this sense, our result represents an improvement and refinement of the known results.

We recall the main notations and definitions that will be used in the sequel.

A function $f: C \times C \rightarrow R$ is said to be strongly monotone on $C$ with modulus $\gamma>0$, if and only if

$$
f(x, y)+f(y, x) \leq-\gamma\|x-y\|^{2}, \quad \forall x, y \in C .
$$

A function $h: C \rightarrow R$ is said to be strongly convex on $C$ with modulus $\beta(\beta \geq 0)$, if and only if

$$
\begin{aligned}
h(\lambda x+(1-\lambda) y) \leq & \lambda h(x)+(1-\lambda) h(y) \\
& -\frac{\beta}{2}[\lambda(1-\lambda)]\|x-y\|^{2}, \\
& \forall x, y \in C, \lambda \in[0,1] .
\end{aligned}
$$

If $h$ is differentiable, then $h$ is strongly convex on $C$ with modulus $\beta(\beta \geq 0)$, if and only if

$$
h(x)-h(y) \geq\langle\nabla h(y), x-y\rangle+\frac{\beta}{2}\|x-y\|^{2}, \quad \forall x, y \in C .
$$

A function $h: C \rightarrow R$ is said to be Lipschitz continuous on $C$ with modulus $L(L>0)$, if and only if

$$
\|h(y)-h(x)\| \leq L\|y-x\|, \quad \forall x, y \in C .
$$

Usually, we need there to be at least one solution for equilibrium problems. In particular, it is true when $C$ is compact.

Proposition 1 (existence of equilibrium (see [19])). Suppose $C$ is nonempty compact convex and $f(x, y)$ is jointly lower semicontinuous, separately continuous in $x$, and convex in $y$. Then (EP) admits at least one solution.

This paper is organized as follows. In Section 2, we introduce some algorithms. In particular, we will give a predictor-corrector algorithmic frame. We present some convergence analysis under perfect and imperfect foresight in Section 3. Section 4 is devoted to an application: we focus on the particular case variational inequalities problem (VIP) of (EP) mentioned above and we apply our results in these frameworks and the predictor-corrector algorithm is applied to (VIP). The paper ends with some concluding remarks.

\section{Main Algorithm}

Most of the algorithms developed for solving EP can be derived from equivalent formulations of the equilibrium problem. We will focus our attention on fixed-point formulations of EP: we will show that such formulations lead to a generalization of the methods developed by Cohen for variational inequalities and optimization problems.

Let us recall the following preliminary result which states the above mentioned equivalent formulation of EP.

Lemma 2. Suppose that $f(x, x)=0$, for all $x \in C$. Then the following statements are equivalent:

(a) there exists $x^{*} \in C$, s.t. $f\left(x^{*}, y\right) \geq 0$, for all $y \in C$;

(b) $x^{*} \in C$ is a solution of the problem

$$
\min _{x \in C} f\left(x^{*}, x\right) \text {. }
$$

We can define the following general iterative algorithm framework.

Algorithm 3. Consider the following.

Step 1. Set $k=0, x^{0} \in C$.

Step 2. Denote by $x^{k+1}$ the solution of the problem: $\min _{y \in C} f\left(x^{k}, y\right)$.

Step 3. If $\left\|x^{k}-x^{k+1}\right\|^{2}<\delta$, for some fixed $\delta>0$, then stop; otherwise let $k=k+1$ and go to Step 2 .

Unfortunately, in most of the cases, it is not possible to apply the previous algorithm directly to the equilibrium problems, for the previous algorithm may cause instabilities in the iterate process. So it is necessary to introduce an auxiliary equilibrium problem, which is equivalent to the equilibrium problem.

Proposition 4. Let $f(x, y)$ be a convex differentiable function with respect to $y$ at $x=x^{*}$ and $\varepsilon>0$. Let $H(x, y): C \times C \rightarrow$ $R$ be a nonnegative, differentiable function on the convex set $C$ with respect to $y$ and such that

(i) $H(x, x)=0$, for all $x \in C$;

(ii) $H_{y}^{\prime}(x, x)=0$, for all $x \in C$. 
Then $x^{*}$ is a solution of EP if and only if it is a solution of the auxiliary equilibrium problem $(A E P)$ :

$$
\text { finding } x^{*} \in C, \quad \text { s.t. } \varepsilon f\left(x^{*}, y\right)+H\left(x^{*}, y\right) \geq 0, \quad \forall y \in C \text {. }
$$

Proof. It is easy to know that if $x^{*}$ is a solution of EP, then it is also a solution of AEP.

Vice versa, let $x^{*}$ be a solution of AEP. Then $x^{*}$ is a minimum point of the problem

$$
\min _{x \in K}\left[\varepsilon f\left(x^{*}, y\right)+H\left(x^{*}, y\right)\right] \text {. }
$$

Because $K$ is convex then $x^{*}$ is an optimal solution for (6) if and only if

$$
\left\langle\varepsilon f_{y}^{\prime}\left(x^{*}, x^{*}\right)+H_{y}^{\prime}\left(x^{*}, x^{*}\right), x^{*}-y\right\rangle \geq 0, \quad \forall y \in K,
$$

so that

$$
\left\langle\varepsilon f_{y}^{\prime}\left(x^{*}, x^{*}\right), x^{*}-y\right\rangle \geq 0, \quad \forall y \in K
$$

Dividing by $\varepsilon$, we obtain that (8) implies, by the convexity of $f\left(x^{*}, \cdot\right)$, that

$$
f\left(x^{*}, y\right) \geq f\left(x^{*}, x^{*}\right)=0, \quad \forall y \in K
$$

Remark 5. Suppose $h: C \rightarrow R$ is a strongly convex differentiable function; denote $H(x, y)=h(y)-h(x)-$ $\langle\nabla h(x), y-x\rangle$, for all $x, y \in C$. We have

$$
\begin{aligned}
H(x, y) & =h(y)-h(x)-\langle\nabla h(x), y-x\rangle \\
& \geq \frac{\beta}{2}\|x-y\|^{2} \geq 0 ; \\
H(x, x) & =0 ; \\
H_{y}^{\prime}(x, x) & =0 .
\end{aligned}
$$

That is, $H(x, y)$ satisfies Proposition 4.

Applying Algorithm 3 to the AEP, we obtain the following iterative method.

Algorithm 6. Consider the following.

Step 1. Set $k=0, x^{0} \in C$.

Step 2. Denote by $x^{k+1}$ the solution of the problem: $\min _{y \in C}\left\{\varepsilon f\left(x^{k}, y\right)+H\left(x^{k}, y\right)\right\}$.

Step 3. If $\left\|x^{k}-x^{k+1}\right\|^{2}<\delta$, for some fixed $\delta>0$, then stop; otherwise let $k=k+1$ and go to Step 2 .

Most papers about EP only study the existence of EP's solution. In this paper, we will give a predictor-corrector method to solve the equilibrium problems.
Definition 7. Let $\mu \in R \backslash\{0\}$ and $x \in C$. A convex function $\bar{f}(x, \cdot): C \rightarrow R$ is a $\mu$-approximation of $f(x, \cdot)$ at $x$, if $\bar{f}(x, \cdot) \leq f(x, \cdot)$ on $C$ and $f(x, \bar{y}) \leq \mu \bar{f}(x, \bar{y})$, where $\bar{y}=$ $\min _{y \in C}\left\{\varepsilon \bar{f}\left(x^{k}, y\right)+H\left(x^{k}, y\right)\right\}$.

Remark 8. According to the above, we extend the coefficient of approximate function from $\mu \in(0,1]$ in $[20]$ to $\mu \in R \backslash\{0\}$, which is a more generic case.

Now, we describe the framework of predictor-corrector algorithm as follows.

Algorithm 9. Let $\alpha_{k} \geq \alpha>0, \varepsilon_{k}>0$, for all $k \in N$.

Step 1. Let $k=0, x^{0} \in C$.

Step 2. Find $\mu$-approximation of $f(x, \cdot)$ at $x, \bar{f}(x, \cdot)$ by predictor-corrector method. Let

$$
\begin{aligned}
x^{k+} & =\min _{y \in C}\left\{\alpha_{k} \bar{f}\left(x^{k}, y\right)+H\left(x^{k}, y\right)\right\} ; \\
x^{k+1} & \in \varepsilon_{k}-\min _{y \in C}\left\{\alpha_{k} f\left(x^{k+}, y\right)+H\left(x^{k+}, y\right)\right\} .
\end{aligned}
$$

Step 3. If $\left\|x^{k}-x^{k+1}\right\|^{2}<\delta$, for some fixed $\delta>0$, then stop; otherwise let $k=k+1$ and go to Step 2 .

Remark 10. In Algorithm 9, each stage of computation requires two proximal steps. In Step $2, x^{k+}$ is served to predict the next point; the other $x^{k+1}$ helps to correct the new prediction.

\section{Convergence Analysis}

In this section, we will give some convergence results about the algorithm.

Definition 11. In Algorithm 9, if $x^{k+1}=x^{k+}, x^{k+}$ is called a perfect foresight point of $x^{k+1}$; otherwise $x^{k+}$ is an imperfect foresight point of $x^{k+1}$.

Next we give the convergence result under perfect foresight, which has been stated in [20].

Proposition 12 (see [20]). Assume that there exist numbers $r, c, d>0$ and a nonnegative function $g: C \times C \rightarrow R$ such that, for all $x, y, z \in C$,

(i) $f(x, y) \geq 0 \Rightarrow f(y, x) \leq-r g(y, x)$;

(ii) $f(x, z)-f(y, z)-f(x, y) \leq c g(x, y)+d\|z-y\|^{2}$.

If the sequence $\left\{\alpha_{k}\right\}_{k \in N}$ is nonincreasing and $\alpha_{k} \leq \beta \mu / 2 d$ for all $k \in N$ and if $c / \mu \leq \mu \leq 1$, then the sequence $\left\{x_{k}\right\}_{k \in N}$ generated by the predictor-corrector algorithm is bounded and $\lim _{k \rightarrow \infty}\left\|x^{k}-x^{k+1}\right\|=0$.

Proposition 13 (see [20]). Assume that $\alpha_{k} \geq \alpha>0$ for all $k \epsilon$ $N$. If the sequence $\left\{x_{k}\right\}_{k \in N}$ generated by the predictor-corrector 
algorithm is bounded and $\lim _{k \rightarrow \infty}\left\|x^{k}-x^{k+1}\right\|=0$, then every limit point of $\left\{x^{k}\right\}_{k \in N}$ is a solution of problem (EP).

At the same time, respective to convergence under imperfect foresight, we first give some denotations and results.

By the previous introduction, we have

$$
\begin{aligned}
& x^{k+}=\arg \min _{y \in C}\left\{\alpha_{k} \bar{f}\left(x^{k}, y\right)+h(y)\right.\left.-h\left(x^{k}\right)-\left\langle\nabla h\left(x^{k}\right), y\right\rangle\right\} ; \\
& x^{k+1} \in \varepsilon_{k}-\arg \min _{y \in C}\left\{\alpha_{k} f\left(x^{k+}, y\right)\right. \\
&\left.+h(y)-h\left(x^{k+}\right)-\left\langle\nabla h\left(x^{k+}\right), y\right\rangle\right\} .
\end{aligned}
$$

Using (12) and (13), we get

$$
\begin{aligned}
& \left\langle\nabla h\left(x^{k}\right)-\nabla h\left(x^{k+}\right), y-x^{k+}\right\rangle \\
& \quad \leq \alpha_{k}\left\{\bar{f}\left(x^{k}, y\right)-\bar{f}\left(x^{k}, x^{k+}\right)\right\}, \quad \forall y \in C, \\
& \left\langle\nabla h\left(x^{k+}\right)-\nabla h\left(x^{k+1}\right), y-x^{k+1}\right\rangle \\
& \quad \leq \alpha_{k}\left\{f\left(x^{k+}, y\right)-f\left(x^{k+}, x^{k+1}\right)+\varepsilon_{k}\right\}, \quad \forall y \in C .
\end{aligned}
$$

Arranging (15), we have

$$
\begin{aligned}
\left\langle\nabla h\left(x^{k}\right)-\nabla h\left(x^{k+1}\right), y-x^{k+1}\right\rangle \\
\leq \alpha_{k}\left\{f\left(x^{k+}, y\right)-f\left(x^{k+}, x^{k+1}\right)+\varepsilon_{k}\right\} \\
+\left\langle\nabla h\left(x^{k}\right)-\nabla h\left(x^{k+}\right), y-x^{k+1}\right\rangle .
\end{aligned}
$$

Let $y=x^{*}$ in (14) and (16); then, adding them, we can get

$$
\begin{aligned}
& \left\langle\nabla h\left(x^{k}\right)-\nabla h\left(x^{k+1}\right), x^{*}-x^{k+1}\right\rangle \\
& \leq \alpha_{k}\left\{f\left(x^{k+}, x^{*}\right)-f\left(x^{k+}, x^{k+1}\right)+\bar{f}\left(x^{k}, x^{*}\right)\right. \\
& \left.-\bar{f}\left(x^{k}, x^{k+}\right)+\varepsilon_{k}\right\} \\
& +\left\langle\nabla h\left(x^{k}\right)-\nabla h\left(x^{k+}\right), x^{k+}-x^{k+1}\right\rangle \\
& \leq \alpha_{k}\left\{f\left(x^{k+}, x^{*}\right)-f\left(x^{k+}, x^{k+1}\right)\right. \\
& \left.+f\left(x^{k}, x^{*}\right)-\frac{1}{\mu} f\left(x^{k}, x^{k+}\right)+\varepsilon_{k}\right\} \\
& +\left\langle\nabla h\left(x^{k}\right)-\nabla h\left(x^{k+}\right), x^{k+}-x^{k+1}\right\rangle, \\
& \left(\bar{f}\left(x^{k}, \cdot\right) \leq f\left(x^{k}, \cdot\right)\right. \\
& \text { on } \left.C, f\left(x^{k}, x^{k+}\right) \leq \mu \bar{f}\left(x^{k}, x^{k+}\right) \text { at } x^{k+}\right) \text {. }
\end{aligned}
$$

Assumption 14. Assume that there exist $r, c, d>0$ and $r \geq$ $\max \{d+(4 c / \mu)+(2 L / \alpha) ;(5 c / \mu)+(2 L / \alpha)\}$, for all $x, y, z \in C$. Consider the following:

(i) $f(x, y) \geq 0 \Rightarrow f(y, x) \leq-r\|x-y\|^{2}$;

(ii) $f(x, z)-f(y, z)-f(x, y) \leq c\|x-y\|^{2}+d\|z-y\|^{2}$.

We denote

$$
\begin{aligned}
& A_{k}=2 \mu \alpha_{k} c+2 \alpha_{k} d+\mu L ; \\
& B_{k}=2 \mu \alpha_{k} c+2 \alpha_{k} d+3 \mu L+4 \alpha_{k} c ; \\
& C_{k}=\frac{2 \alpha_{k} d}{\mu} ; \\
& E_{k}=\min \left\{\frac{r-d}{4}-\frac{c}{\mu}-\frac{L}{2 \alpha_{k}}, \frac{\beta-B_{k}}{4 \mu \alpha_{k}}, \frac{\alpha_{k} r \mu-5 \alpha_{k} c-2 \mu L}{4 \mu \alpha_{k}}\right\} .
\end{aligned}
$$

It is convenient to prove the following theorem.

Theorem 15. Assume that the function $f(x, y)$ satisfies Assumption 14 and $\beta \geq \max \left\{A_{k}, B_{k}, C_{k}\right\}, \varepsilon_{k} \leq E_{k}\left\|x^{k}-x^{k+}\right\|^{2}$; then the sequence $\left\{x^{k}\right\}_{k \in N}$ generated by the predictor-corrector methods is bounded and $\lim _{k \rightarrow \infty}\left\|x^{k}-x^{k+1}\right\|=0$.

Proof. Let $x^{*}$ be a solution of (EP) and consider for each $k \epsilon$ $N$ the Lyapunov function $\Gamma^{k}: C \times C \rightarrow R$ defined for all $y, z \in C$ :

$$
\Gamma^{k}(y, z)=h(z)-h(y)-\langle\nabla h(y), z-y\rangle+\frac{\alpha_{k}}{\mu} f(z, y) .
$$

Since $h$ is strongly convex on $C$ with modulus $\beta$, we can easily obtain that, for all $x^{k} \in C$,

$$
\Gamma^{k}\left(x^{k}, x^{*}\right) \geq \frac{\beta}{2}\left\|x^{k}-x^{*}\right\|^{2} .
$$

Consider the following relation:

$$
\begin{aligned}
\Gamma^{k+1} & \left(x^{k+1}, x^{*}\right)-\Gamma^{k}\left(x^{k}, x^{*}\right) \\
\leq & h\left(x^{k}\right)-h\left(x^{k+1}\right)+\left\langle\nabla h\left(x^{k}\right), x^{k+1}-x^{k}\right\rangle \\
& +\left\langle\nabla h\left(x^{k}\right)-\nabla h\left(x^{k+1}\right), x^{*}-x^{k+1}\right\rangle \\
& +\frac{\alpha_{k}}{\mu}\left\{f\left(x^{*}, x^{k+1}\right)-f\left(x^{*}, x^{k}\right)\right\} \\
= & S_{1}+S_{2}+S_{3},
\end{aligned}
$$

where

$$
\begin{aligned}
& S_{1}=h\left(x^{k}\right)-h\left(x^{k+1}\right)+\left\langle\nabla h\left(x^{k}\right), x^{k+1}-x^{k}\right\rangle ; \\
& S_{2}=\left\langle\nabla h\left(x^{k}\right)-\nabla h\left(x^{k+1}\right), x^{*}-x^{k+1}\right\rangle ; \\
& S_{3}=\frac{\alpha_{k}}{\mu}\left\{f\left(x^{*}, x^{k+1}\right)-f\left(x^{*}, x^{k}\right)\right\} .
\end{aligned}
$$


For $S_{1}$, we can easily get the following from the strong convexity of $h$ :

$$
S_{1} \leq-\frac{\beta}{2}\left\|x^{k+1}-x^{k}\right\|^{2} .
$$

For $S_{2}$, we derive the following from (17):

$$
\begin{aligned}
S_{2} \leq \alpha_{k}\left\{f\left(x^{k+}, x^{*}\right)-f\left(x^{k+}, x^{k+1}\right)+f\left(x^{k}, x^{*}\right)\right. \\
\left.\quad-\frac{1}{\mu} f\left(x^{k}, x^{k+}\right)+\varepsilon_{k}\right\} \\
+\left\langle\nabla h\left(x^{k}\right)-\nabla h\left(x^{k+}\right), x^{k+}-x^{k+1}\right\rangle .
\end{aligned}
$$

Then

$$
\begin{aligned}
S_{2}+S_{3} \leq & \alpha_{k}\left\{f\left(x^{k+}, x^{*}\right)-f\left(x^{k+}, x^{k+1}\right)+f\left(x^{k}, x^{*}\right)\right. \\
& \left.-\frac{1}{\mu} f\left(x^{k}, x^{k+}\right)+\varepsilon_{k}\right\} \\
+ & \frac{\alpha_{k}}{\mu}\left\{f\left(x^{*}, x^{k+1}\right)-f\left(x^{*}, x^{k}\right)\right\} \\
+ & \left\langle\nabla h\left(x^{k}\right)-\nabla h\left(x^{k+}\right), x^{k+}-x^{k+1}\right\rangle \\
= & \alpha_{k}\left\{f\left(x^{k+}, x^{*}\right)-f\left(x^{k+}, x^{k+1}\right)+f\left(x^{k}, x^{*}\right)+\varepsilon_{k}\right\} \\
& +\frac{\alpha_{k}}{\mu}\left\{f\left(x^{k}, x^{k+1}\right)-f\left(x^{k}, x^{k+}\right)\right\} \\
& +\frac{\alpha_{k}}{\mu}\left\{f\left(x^{*}, x^{k+1}\right)-f\left(x^{*}, x^{k}\right)-f\left(x^{k}, x^{k+1}\right)\right\} \\
& +\left\langle\nabla h\left(x^{k}\right)-\nabla h\left(x^{k+}\right), x^{k+}-x^{k+1}\right\rangle .
\end{aligned}
$$
have

For the last term on the right of the above equality, we

$$
\begin{aligned}
& \left\langle\nabla h\left(x^{k}\right)-\nabla h\left(x^{k+}\right), x^{k+}-x^{k+1}\right\rangle \\
& \leq \frac{L}{2}\left\|x^{k}-x^{k+}\right\|^{2}+\frac{L}{2}\left\|x^{k+}-x^{k+1}\right\|^{2} .
\end{aligned}
$$

We can obtain the following from assumption (ii):

$$
\begin{aligned}
& f\left(x^{k+}, x^{*}\right)-f\left(x^{k+}, x^{k+1}\right) \\
& =f\left(x^{k+}, x^{*}\right)-f\left(x^{k+}, x^{k+1}\right)-f\left(x^{k+1}, x^{*}\right)+f\left(x^{k+1}, x^{*}\right) \\
& \leq c\left\|x^{k+}-x^{k+1}\right\|^{2}+(d-r)\left\|x^{k+1}-x^{*}\right\|^{2} .
\end{aligned}
$$

Similarly,

$$
\begin{aligned}
& f\left(x^{*}, x^{k+1}\right)-f\left(x^{*}, x^{k}\right)-f\left(x^{k}, x^{k+1}\right) \\
& \leq c\left\|x^{*}-x^{k}\right\|^{2}+d\left\|x^{k}-x^{k+1}\right\|^{2} ; \\
& f\left(x^{k}, x^{k+1}\right)-f\left(x^{k}, x^{k+}\right) \\
& =f\left(x^{k}, x^{k+1}\right)-f\left(x^{k}, x^{k+}\right) \\
& \quad-f\left(x^{k+}, x^{k+1}\right)+f\left(x^{k+}, x^{k+1}\right) \\
& \leq c\left\|x^{k}-x^{k+}\right\|^{2}+d\left\|x^{k+1}-x^{k+}\right\|^{2}+f\left(x^{k+}, x^{k+1}\right) .
\end{aligned}
$$

Because of $\alpha_{k} f\left(x^{k+}, x^{k+1}\right) \leq-(\beta / 2)\left\|x^{k+1}-x^{k+}\right\|^{2}$, we derive the following from (13):

$$
\begin{aligned}
& \alpha_{k} f\left(x^{k+}, x^{k+1}\right)+h\left(x^{k+1}\right)-h\left(x^{k+}\right) \\
& -\left\langle\nabla h\left(x^{k+}\right), x^{k+1}-x^{k+}\right\rangle \\
& \leq \alpha_{k} \bar{f}\left(x^{k+}, y\right)+h(y)-h\left(x^{k+}\right)-\left\langle\nabla h\left(x^{k+}\right), y-x^{k+}\right\rangle, \\
& \text { In particular, let } y=x^{k+} ; \text { we have } \\
& \alpha_{k} f\left(x^{k+}, x^{k+1}\right)+h\left(x^{k+1}\right)-h\left(x^{k+}\right) \\
& \quad-\left\langle\nabla h\left(x^{k+}\right), x^{k+1}-x^{k+}\right\rangle \leq f\left(x^{k+}, x^{k+}\right)=0 .
\end{aligned}
$$$$
\forall y \in C \text {. }
$$

That is,

$$
\begin{aligned}
& \alpha_{k} f\left(x^{k+}, x^{k+1}\right)+h\left(x^{k+1}\right)-h\left(x^{k+}\right) \\
&-\left\langle\nabla h\left(x^{k+}\right), x^{k+1}-x^{k+}\right\rangle \leq 0 \\
& \Longleftrightarrow \alpha_{k} f\left(x^{k+}, x^{k+1}\right) \leq-h\left(x^{k+1}\right)+h\left(x^{k+}\right) \\
&+\left\langle\nabla h\left(x^{k+}\right), x^{k+1}-x^{k+}\right\rangle \\
& \leq-\frac{\beta}{2}\left\|x^{k+1}-x^{k+}\right\|^{2} .
\end{aligned}
$$

Hence,

$$
\alpha_{k} f\left(x^{k+}, x^{k+1}\right) \leq-\frac{\beta}{2}\left\|x^{k+1}-x^{k+}\right\|^{2} .
$$

Finally, we obtain

$$
\begin{gathered}
f\left(x^{k}, x^{k+1}\right)-f\left(x^{k}, x^{k+}\right) \\
\leq c\left\|x^{k}-x^{k+}\right\|^{2}+d\left\|x^{k+1}-x^{k+}\right\|^{2} \\
-\frac{\beta}{2 \alpha_{k}}\left\|x^{k+1}-x^{k+}\right\|^{2} .
\end{gathered}
$$


So

$$
\begin{aligned}
& S_{1}+S_{2}+S_{3} \\
& \leq \alpha_{k}\left\{c\left\|x^{k+}-x^{k+1}\right\|^{2}+(d-r)\left\|x^{k+1}-x^{*}\right\|^{2}\right. \\
& \left.-r\left\|x^{k}-x^{*}\right\|^{2}+\varepsilon_{k}\right\} \\
& +\frac{\alpha_{k}}{\mu}\left\{c\left\|x^{k}-x^{k+}\right\|^{2}+d\left\|x^{k+1}-x^{k+}\right\|^{2}\right. \\
& \left.\quad-\frac{\beta}{2 \alpha_{k}}\left\|x^{k+1}-x^{k+}\right\|^{2}\right\} \\
& +\frac{\alpha_{k}}{\mu}\left\{c\left\|x^{*}-x^{k}\right\|^{2}+d\left\|x^{k}-x^{k+1}\right\|^{2}\right\} \\
& +\frac{L}{2}\left\|x^{k}-x^{k+}\right\|^{2}+\frac{L}{2}\left\|x^{k+}-x^{k+1}\right\|^{2} \\
& -\frac{\beta}{2}\left\|x^{k+1}-x^{k}\right\|^{2} .
\end{aligned}
$$

Arrange the previous inequality relation; we can get

$S_{1}+S_{2}+S_{3}$

$$
\begin{aligned}
\leq & -\left(\frac{\beta}{2}-\frac{\alpha_{k} d}{\mu}\right)\left\|x^{k}-x^{k+1}\right\|^{2}+\left(\alpha_{k} c+\frac{\alpha_{k} d}{\mu}+\frac{L}{2}-\frac{\beta}{2 \mu}\right) \\
& \times\left\|x^{k+}-x^{k+1}\right\|^{2}+\alpha_{k}(d-r)\left\|x^{k+1}-x^{*}\right\|^{2}+\alpha_{k}\left(\frac{c}{\mu}-r\right) \\
& \times\left\|x^{k}-x^{*}\right\|^{2}+\left(\frac{\alpha_{k} c}{\mu}+\frac{L}{2}\right)\left\|x^{k}-x^{k+}\right\|^{2}+\alpha_{k} \varepsilon_{k} .
\end{aligned}
$$

Under the condition of $(\beta / 2)-\left(\alpha_{k} d / \mu\right)>0$, in order to obtain $S_{1}+S_{2}+S_{3} \leq 0$, we only need to prove the following result:

$$
\begin{aligned}
& \left(\alpha_{k} c+\frac{\alpha_{k} d}{\mu}+\frac{L}{2}-\frac{\beta}{2 \mu}\right)\left\|x^{k+}-x^{k+1}\right\|^{2} \\
& +\alpha_{k}(d-r)\left\|x^{k+1}-x^{*}\right\|^{2} \\
& +\alpha_{k}\left(\frac{c}{\mu}-r\right)\left\|x^{k}-x^{*}\right\|^{2}+\left(\frac{\alpha_{k} c}{\mu}+\frac{L}{2}\right) \\
& \times\left\|x^{k}-x^{k+}\right\|^{2}+\alpha_{k} \varepsilon_{k} \leq 0 .
\end{aligned}
$$

That is,

$$
\begin{aligned}
& \left(\frac{\alpha_{k} c}{\mu}+\frac{L}{2}\right)\left\|x^{k}-x^{k+}\right\|^{2} \\
& \leq \alpha_{k}(r-d)\left\|x^{k+1}-x^{*}\right\|^{2}+\alpha_{k}\left(r-\frac{c}{\mu}\right)\left\|x^{k}-x^{*}\right\|^{2} \\
& \quad+\left(\frac{\beta}{2 \mu}-\alpha_{k} c-\frac{\alpha_{k} d}{\mu}-\frac{L}{2}\right)\left\|x^{k+}-x^{k+1}\right\|^{2}
\end{aligned}
$$

(1) When $\mu d \geq c$,

$$
\begin{aligned}
& \alpha_{k}(r-d)\left\|x^{k+1}-x^{*}\right\|^{2}+\alpha_{k}\left(r-\frac{c}{\mu}\right)\left\|x^{k}-x^{*}\right\|^{2} \\
& \geq \alpha_{k}(r-d)\left\{\left\|x^{k+1}-x^{*}\right\|^{2}+\left\|x^{k}-x^{*}\right\|^{2}\right\} \\
& \geq \frac{\alpha_{k}(r-d)}{2}\left\|x^{k+1}-x^{k}\right\|^{2} .
\end{aligned}
$$

Then

$$
\begin{gathered}
\alpha_{k}(r-d)\left\|x^{k+1}-x^{*}\right\|^{2}+\alpha_{k}\left(r-\frac{c}{\mu}\right)\left\|x^{k}-x^{*}\right\|^{2} \\
+\left(\frac{\beta}{2 \mu}-\alpha_{k} c-\frac{\alpha_{k} d}{\mu}-\frac{L}{2}\right)\left\|x^{k+}-x^{k+1}\right\|^{2} \\
\geq \frac{\alpha_{k}(r-d)}{2}\left\|x^{k+1}-x^{k}\right\|^{2}+\frac{\beta-A_{k}}{2 \mu}\left\|x^{k+}-x^{k+1}\right\|^{2} .
\end{gathered}
$$

We discuss in two cases.

$\left(1^{\circ}\right)$ If $\left(\beta-A_{k}\right) / 2 \mu \geq \alpha_{k}(r-d) / 2$,

$$
\begin{aligned}
& \frac{\alpha_{k}(r-d)}{2}\left\|x^{k+1}-x^{k}\right\|^{2}+\frac{\beta-A_{k}}{2 \mu}\left\|x^{k+}-x^{k+1}\right\|^{2} \\
& \geq \frac{\alpha_{k}(r-d)}{2}\left\{\left\|x^{k+}-x^{k+1}\right\|^{2}+\left\|x^{k+1}-x^{k}\right\|^{2}\right\} \\
& \geq \frac{\alpha_{k}(r-d)}{4}\left\|x^{k+}-x^{k}\right\|^{2} .
\end{aligned}
$$

So

$$
\begin{aligned}
S_{1} & +S_{2}+S_{3} \\
\leq & -\left(\frac{\beta}{2}-\frac{\alpha_{k} d}{\mu}\right)\left\|x^{k}-x^{k+1}\right\|^{2}-\left[\frac{\alpha_{k}(r-d)}{4}-\frac{\alpha_{k} c}{\mu}-\frac{L}{2}\right] \\
& \times\left\|x^{k}-x^{k+}\right\|^{2}+\alpha_{k} \varepsilon_{k} .
\end{aligned}
$$

We know that

$$
\frac{\beta}{2}-\frac{\alpha_{k} d}{\mu}>0, \quad \frac{\alpha_{k}(r-d)}{4}-\frac{\alpha_{k} c}{\mu}-\frac{L}{2}>0,
$$

$\alpha_{k} \varepsilon_{k} \leq\left[\frac{\alpha_{k}(r-d)}{4}-\frac{\alpha_{k} c}{\mu}-\frac{L}{2}\right]\left\|x^{k}-x^{k+1}\right\|^{2}+\alpha_{k} \varepsilon_{k}$.

Finally, we get $S_{1}+S_{2}+S_{3} \leq 0$; it follows that $\left\{\Gamma^{k}\left(x^{k}, x^{*}\right)\right\}_{k \in N}$ is a nonincreasing sequence. By (21), we know that $\left\{\Gamma^{k}\left(x^{k}, x^{*}\right)\right\}_{k \in N}$ is bounded below by 0 . Hence, $\left\{\Gamma^{k}\left(x^{k}, x^{*}\right)\right\}_{k \in N}$ converges in $R$ and $\left\{x^{k}\right\}_{k \in N}$ is bounded. Passing to the limit in (42), then $\left\|x^{k}-x^{k+1}\right\|^{2} \rightarrow 0(k \rightarrow$ $\infty)$. 
$\left(2^{\circ}\right)$ If $\left(\beta-A_{k}\right) / 2 \mu<\alpha_{k}(r-d) / 2$,

$$
\begin{aligned}
S_{1}+S_{2} & +S_{3} \\
\leq & -\left(\frac{\beta}{2}-\frac{\alpha_{k} d}{\mu}\right)\left\|x^{k}-x^{k+1}\right\|^{2} \\
& -\left[\frac{\beta-A_{k}}{4 \mu}-\frac{\alpha_{k} c}{\mu}-\frac{L}{2}\right]\left\|x^{k}-x^{k+}\right\|^{2}+\alpha_{k} \varepsilon_{k} .
\end{aligned}
$$

Similarly to $\left(1^{\circ}\right)$, we can obtain the result.

(2) When $\mu d<c$,

$$
\begin{gathered}
\alpha_{k}(r-d)\left\|x^{k+1}-x^{*}\right\|^{2}+\alpha_{k}\left(r-\frac{c}{\mu}\right)\left\|x^{k}-x^{*}\right\|^{2} \\
\geq \alpha_{k}\left(r-\frac{c}{\mu}\right)\left\{\left\|x^{k+1}-x^{*}\right\|^{2}+\left\|x^{k}-x^{*}\right\|^{2}\right\} \\
\geq \frac{\alpha_{k}(r-c / \mu)}{2}\left\|x^{k+1}-x^{k}\right\|^{2} .
\end{gathered}
$$

Likewise, we also discuss in two cases.

$\left(1^{\circ}\right)$ When $\left(\beta-A_{k}\right) / 2 \mu \geq \alpha_{k}(r-c / \mu) / 2$,

$$
\begin{aligned}
& S_{1}+S_{2}+S_{3} \\
& \leq-\left(\frac{\beta}{2}-\frac{\alpha_{k} d}{\mu}\right)\left\|x^{k}-x^{k+1}\right\|^{2}-\left[\frac{\alpha_{k}(r-c / \mu)}{4}-\frac{\alpha_{k} c}{\mu}-\frac{L}{2}\right] \\
& \quad \times\left\|x^{k}-x^{k+}\right\|^{2}+\alpha_{k} \varepsilon_{k} .
\end{aligned}
$$

Similarly to the proof of (1), we omit the process and get the conclusion.

$\left(2^{\circ}\right)$ When $\left(\beta-A_{k}\right) / 2 \mu<\alpha_{k}(r-c / \mu) / 2$.

Similar to the proof of (1), we omit the process and get the conclusion.

Theorem 16. Assume that $\alpha_{k} \geq \alpha>0$ for all $k \in N$. If the sequence $\left\{x_{k}\right\}_{k \in N}$ generated by the predictor-corrector algorithm is bounded and $\lim _{k \rightarrow \infty}\left\|x^{k}-x^{k+1}\right\|=0$, then every limit point of $\left\{x^{k}\right\}_{k \in N}$ is a solution of equilibrium problem.

Proof. Let $x^{*}$ be the limiting point of $\left\{x^{k}\right\}_{k \in N}$ and denote by $\left\{x^{k}\right\}_{k \in K \subset N}$ some subsequence converging to $x^{*}$. According to

$$
\begin{aligned}
& x^{k+} \\
& =\arg \min _{y \in C}\left\{\alpha_{k} \bar{f}\left(x^{k}, y\right)+h(y)-h\left(x^{k}\right)-\left\langle\nabla h\left(x^{k}\right), y\right\rangle\right\} \\
& =\arg \min _{y \in C} \alpha_{k} \bar{f}\left(x^{k}, y\right),
\end{aligned}
$$

we obtain

$$
\begin{aligned}
0 \leq & \bar{f}\left(x^{k}, y\right)-\bar{f}\left(x^{k}, x^{k+}\right) \\
\leq & f\left(x^{k}, y\right)-\frac{1}{\mu} f\left(x^{k}, x^{k+}\right) \Longleftrightarrow \\
0 \leq & (\mu-1) f\left(x^{k}, y\right)+f\left(x^{k}, y\right)-f\left(x^{k}, x^{k+}\right) \\
= & (\mu-1) f\left(x^{k}, y\right)+c\left\|x^{k}-x^{k+}\right\|^{2} \\
& +d\left\|y-x^{k+}\right\|^{2}+f\left(x^{k+}, y\right), \quad \forall y \in C .
\end{aligned}
$$

In particular, we set $y=x^{k+1}$; then

$$
\begin{aligned}
0 \leq & (\mu-1) f\left(x^{k}, x^{k+1}\right)+c\left\|x^{k}-x^{k+}\right\|^{2} \\
& +d\left\|x^{k+1}-x^{k+}\right\|^{2}+f\left(x^{k+}, x^{k+1}\right) \\
\leq & (\mu-1) f\left(x^{k}, x^{k+1}\right)+c\left\|x^{k}-x^{k+}\right\|^{2}+d\left\|x^{k+1}-x^{k+}\right\|^{2} \\
& -\frac{\beta}{2 \alpha_{k}}\left\|x^{k+}-x^{k+1}\right\|^{2} \\
= & (\mu-1) f\left(x^{k}, x^{k+1}\right)+c\left\|x^{k}-x^{k+}\right\|^{2} \\
& +\left(d-\frac{\beta}{2 \alpha_{k}}\right)\left\|x^{k+}-x^{k+1}\right\|^{2} \\
\leq & (\mu-1) f\left(x^{k}, x^{k+1}\right)+\frac{c}{2}\left\|x^{k+1}-x^{k}\right\|^{2} \\
& +\frac{c}{2}\left\|x^{k+}-x^{k+1}\right\|^{2}+\left(d-\frac{\beta}{2 \alpha_{k}}\right)\left\|x^{k+}-x^{k+1}\right\|^{2} \\
= & (\mu-1) f\left(x^{k}, x^{k+1}\right)+\frac{c}{2}\left\|x^{k+1}-x^{k}\right\|^{2} \\
& +\left(d-\frac{\beta}{2 \alpha_{k}}+\frac{c}{2}\right)\left\|x^{k+}-x^{k+1}\right\|^{2} .
\end{aligned}
$$

That is,

$$
\begin{aligned}
& \left(\frac{\beta}{2 \alpha_{k}}-d-\frac{c}{2}\right)\left\|x^{k+}-x^{k+1}\right\|^{2} \\
& \quad \leq(\mu-1) f\left(x^{k}, x^{k+1}\right)+\frac{c}{2}\left\|x^{k+1}-x^{k}\right\|^{2} .
\end{aligned}
$$

Passing to the limit in (50) as $k \rightarrow \infty$, then

$$
f\left(x^{k}, x^{k+1}\right) \longrightarrow f\left(x^{*}, x^{*}\right)=0, \quad\left\|x^{k+1}-x^{k}\right\|^{2} \longrightarrow 0 .
$$

At the same time, $\beta>\alpha_{k}(2 d+c)$, so $\left\|x^{k+}-x^{k+1}\right\|^{2} \rightarrow 0$. From $\left\|x^{k}-x^{k+1}\right\|^{2} \rightarrow 0$, we have $\left\|x^{k+}-x^{k}\right\|^{2} \rightarrow 0$. Moreover, $\left\|x^{*}-x^{k}\right\|^{2} \rightarrow 0$; we get $\left\|x^{k+}-x^{*}\right\|^{2} \rightarrow 0$. 
Due to $\bar{f}\left(x^{k}, x^{k+}\right) \leq f\left(x^{k}, x^{k+}\right)$ at $x^{k}$ and $\mu \bar{f}\left(x^{k}, x^{k+}\right) \geq$ $f\left(x^{k}, x^{k+}\right)$, then

$$
\frac{1}{\mu} f\left(x^{k}, x^{k+}\right) \leq \bar{f}\left(x^{k}, x^{k+}\right) \leq f\left(x^{k}, x^{k+}\right) .
$$

For all $k \in K$, when $k \rightarrow \infty$, we have

$$
x^{k} \longrightarrow x^{*}, \quad x^{k+} \longrightarrow x^{*} .
$$

In addition, $f$ is continuous; we have

$$
f\left(x^{k}, x^{k+}\right) \longrightarrow f\left(x^{*}, x^{*}\right)=0, \quad(k \longrightarrow \infty) .
$$

Hence

$$
f\left(x^{k}, x^{k+}\right) \longrightarrow 0, \quad(k \longrightarrow \infty) .
$$

Since $x^{k+}=\arg \min _{y \in C}\left\{\alpha_{k} \bar{f}\left(x^{k}, y\right)+h(y)-h\left(x^{k}\right)-\right.$ $\left.\left\langle\nabla h\left(x^{k}\right), y\right\rangle\right\}$, we have

$$
0 \in \partial\left\{\alpha_{k} \bar{f}\left(x^{k}, \cdot\right)+\psi_{C}\right\}\left(x^{k+}\right)-\nabla h\left(x^{k}\right)+\nabla h\left(x^{k+}\right) .
$$

That is,

$$
\nabla h\left(x^{k}\right)-\nabla h\left(x^{k+}\right) \in \partial\left\{\alpha_{k} \bar{f}\left(x^{k}, \cdot\right)+\psi_{C}\right\}\left(x^{k+}\right),
$$

where $\varphi_{C}$ denotes the indicate function of the set $C$. Using the definition of subdifferential, we get

$$
\begin{aligned}
& \bar{f}\left(x^{k}, y\right)-\bar{f}\left(x^{k}, x^{k+}\right) \\
& \quad \geq \frac{1}{\alpha_{k}}\left\langle\nabla h\left(x^{k}\right)-\nabla h\left(x^{k+}\right), y-x^{k+}\right\rangle, \quad \forall y \in C .
\end{aligned}
$$

Applying the Cauchy-Schwarz inequality and the properties $\bar{f}\left(x^{k}, \cdot\right) \leq f\left(x^{k}, \cdot\right)$ and that $\nabla h$ is Lipschitz continuous on $C$ with constant $L$, we have, for all $y \in C$,

$$
\begin{aligned}
& f\left(x^{k}, y\right)-\bar{f}\left(x^{k}, x^{k+}\right) \\
& \geq-\frac{1}{\alpha_{k}}\left\|\nabla h\left(x^{k}\right)-\nabla h\left(x^{k+}\right)\right\|\left\|y-x^{k+}\right\| \\
& \geq-\frac{L}{\alpha_{k}}\left\|x^{k}-x^{k+}\right\|\left\|y-x^{k+}\right\|, \quad \forall y \in C .
\end{aligned}
$$

Take the limit about $k \in N$; we deduce

$$
f\left(x^{*}, y\right) \geq 0, \quad \forall y \in C .
$$

Because $f$ is continuous, when $k \rightarrow \infty$,

$$
\begin{gathered}
f\left(x^{k}, y\right) \longrightarrow f\left(x^{*}, y\right), \quad\left\|x^{k}-x^{k+}\right\| \longrightarrow 0, \\
\left\|y-x^{k+}\right\| \longrightarrow\left\|y-x^{*}\right\| .
\end{gathered}
$$

We finish the proof.

For practical implementation, it is necessary to give a stopping criterion.
Definition 17. Let $\Delta \geq 0$. A point $x^{*}$ is called a $\Delta$-stationary point of problem (EP) if $x^{*} \in C$ and

$$
\exists \gamma \in \partial_{\Delta}\left(f\left(x^{*}, \cdot\right)+\varphi_{C}\right)\left(x^{*}\right), \quad \text { s.t. }\|\gamma\| \leq \Delta .
$$

Proposition 18 (see [20]). Let $x^{k+}=\arg \min _{y \in C}\left\{\alpha_{k} \bar{f}\left(x^{k}\right.\right.$, $\left.y)+h(y)-h\left(x^{k}\right)-\left\langle\nabla h\left(x^{k}\right), y-x^{k}\right\rangle\right\} ; \gamma^{k}=\left(1 / \alpha_{k}\right)\left[\nabla h\left(x^{k}\right)-\right.$ $\left.\nabla h\left(x^{k+}\right)\right]=\left(1 / \alpha_{k}\right)\left[\nabla h\left(x^{k}\right)-\nabla h\left(x^{k+1}\right)\right] ; \delta^{k}=\left\langle\gamma_{k}^{i}, x^{k+}-x^{k}\right\rangle-$ $\bar{f}\left(x^{k}, x^{k+}\right)$.

Then $\delta^{k} \geq 0$ and $\gamma^{k} \in \partial_{\delta^{k}}\left(f\left(x^{k}, \cdot\right)+\psi_{c}\right)\left(x^{k}\right)$.

Theorem 19. Assume that $\alpha_{k} \geq \alpha>0$ for all $k \in N$ and that the assumptions of Theorem 15 hold. Let $\left\{x^{k}\right\}_{k \in N}$ be generated by the predictor-corrector algorithm, then the sequences $\left\{\gamma^{k}\right\}_{k \in N}$ and $\left\{\delta^{k}\right\}_{k \in N}$ converge to zero.

Proof. Here we still discuss in two cases.

(1) Under perfect foresight.

Under perfect foresight, it is easy to get $x^{k+}=x^{k+1}$.

Since $\left\{x^{k}\right\}_{k \in N}$ is infinite, it follows from Theorem 16 that the sequence converges to some solution $x^{*}$ of problem (EP).

On the other hand, for all $k$, we have

$$
\begin{aligned}
0 & \leq\left\|\gamma^{k}\right\| \leq\left\|\frac{\nabla h\left(x^{k}\right)-\nabla h\left(x^{k+}\right)}{\alpha_{k}}\right\| \\
& \leq \frac{L}{\alpha}\left\|x^{k}-x^{k+}\right\|=\frac{L}{\alpha}\left\|x^{k}-x^{k+1}\right\| .
\end{aligned}
$$

Because $\nabla h$ is Lipschitz-continuous with constant $L, \alpha_{k} \geq$ $\alpha>0$.

Since $\lim _{k \rightarrow \infty}\left\|x^{k}-x^{k+1}\right\|=0$, we obtain that the sequence $\left\{\gamma^{k}\right\}_{k \in N}$ converges to zero.

Moreover,

$$
\begin{aligned}
& \left|\left\langle\gamma^{k}, x^{k+}-x^{k}\right\rangle\right| \\
& \leq\left\|\gamma^{k}\right\|\left\|x^{k+}-x^{k}\right\|=\left\|\gamma^{k}\right\|\left\|x^{k+1}-x^{k}\right\| \\
& \Longrightarrow\left\langle\gamma^{k}, x^{k+}-x^{k}\right\rangle \longrightarrow 0, \text { as } k \longrightarrow \infty .
\end{aligned}
$$

Finally, by continuity of $f$, so that when $k \rightarrow \infty$,

$$
f\left(x^{k}, x^{k+1}\right) \longrightarrow f\left(x^{*}, x^{*}\right)=0 ;
$$

$f\left(x^{k}, x^{k+1}\right)=\bar{f}\left(x^{k}, x^{k+1}\right) \rightarrow 0(k \rightarrow \infty)$; that is, $\delta^{k} \rightarrow 0$ $(k \rightarrow \infty)$.

(2) Under imperfect foresight.

We derive that $\lim _{k \rightarrow \infty}\left\|x^{k}-x^{k+}\right\|=0$ in the process of proving Theorem 16.

Hence, the sequence $\left\{\gamma^{k}\right\}_{k \in N}$ converges to zero.

Moreover,

$$
\begin{aligned}
\left|\left\langle\gamma^{k}, x^{k+}-x^{k}\right\rangle\right| & \leq\left\|\gamma^{k}\right\|\left\|x^{k+}-x^{k}\right\| \\
& \Longrightarrow\left\langle\gamma^{k}, x^{k+}-x^{k}\right\rangle \longrightarrow 0,
\end{aligned}
$$


because $f$ is continuous, so, when $k \rightarrow \infty, f\left(x^{k}, x^{k+}\right) \rightarrow$ $f\left(x^{*}, x^{*}\right)=0$.

At the same time,

$$
\bar{f}\left(x^{k}, x^{k+}\right) \leq f\left(x^{k}, x^{k+}\right) \leq \mu \bar{f}\left(x^{k}, x^{k+}\right) .
$$

Hence, $\bar{f}\left(x^{k}, x^{k+}\right) \rightarrow 0$; that is, $\delta^{k} \rightarrow 0(k \rightarrow \infty)$.

Next, we give the predictor-corrector algorithm about the (EP) with stopping criterion.

Algorithm 20 (the predictor-corrector algorithms for (EP)). Let $\alpha_{k} \geq \alpha>0, \varepsilon_{k}>0$, for all $k \in N$.

Step 1. Let $k=0, x^{0} \in C$, and $\delta>0$.

Step 2. Finding a $\mu$-approximation $\bar{f}(x, \cdot)$ of $f(x, \cdot)$ at $x$ by predictor-corrector method, let

$$
\begin{aligned}
& x^{k+}=\min _{y \in C}\left\{\alpha_{k} \bar{f}\left(x^{k}, y\right)+H\left(x^{k}, y\right)\right\} ; \\
& \varepsilon_{k} \leq E_{K}\left\|x^{k}-x^{k+}\right\|^{2} ; \\
& x^{k+1} \in \varepsilon_{k}-\min _{y \in C}\left\{\alpha_{k} f\left(x^{k+}, y\right)+H\left(x^{k+}, y\right)\right\} .
\end{aligned}
$$

Step 3. If $\left\|x^{k}-x^{k+1}\right\|^{2}<\delta$, then stop; otherwise put $k=k+1$ and go to Step 2 .

\section{Application to Variational Inequality Problems}

Variational inequalities theory, which was introduced by Stampacchia [21], provides us with a simple, direct, natural, general, efficient, and unified framework to study a wide class of problems arising in pure and applied sciences. It has been extended and generalized in several directions using innovative and novel techniques for studying a wide class of equilibrium problems arising in financial, economics, transportation, elasticity, and optimization. During the last three decades, there has been considerable activity in the development for solving variational inequalities. For the applications, physical formulation, numerical methods, and other aspects of variational inequalities, see [21-27] and the references therein.

Let $F: C \rightarrow C^{*}$ be a given mapping; variational inequality problems are as follows:

$$
\begin{gathered}
\text { finding an } x^{*} \in C, \\
\text { s.t. }\left\langle F\left(x^{*}\right), y-x^{*}\right\rangle \geq 0, \quad \forall y \in C \text {. }
\end{gathered}
$$

We denote $f(x, y)=\langle F(x), y-x\rangle$; then the problem (EP) is equivalent to the problem (VIP).

Similarly to Assumption 14, we have the following.

Assumption 21. Suppose that there exist $r, c, d>0$ and $r \geq$ $\max \{d, c / \mu\}$, for all $x, y, z \in C$ : (i) $\langle F(x), y-x\rangle \geq 0 \Rightarrow\langle F(y), x-y\rangle \leq-r\|x-y\|^{2}$;

(ii) $\langle F(y)-F(z), x-y\rangle \leq c\|y-z\|^{2}+d\|x-y\|^{2}$.

In the same way, we consider the following two cases: perfect foresight and unperfect foresight cases.

First case is under perfect foresight.

Similar to Propositions 12 and 13, we have the following.

Proposition 22. Assume that there exist $r, c, d>0$ and $a$ nonnegative function $g: C \times C \rightarrow R$ such that, for all $x, y, z \in C$,

(i) $\langle F(x), y-x\rangle \geq 0 \Rightarrow\langle F(y), x-y\rangle \leq-r g(y, x)$;

(ii) $\langle F(y)-F(z), x-y\rangle \leq c g(x, y)+d\|z-y\|^{2}$.

If the sequence $\left\{\alpha_{k}\right\}_{k \in N}$ is nonincreasing and the $\alpha_{k} \leq \beta \mu / 2 d$ for all $k \in N$ and if $c / \mu \leq \mu \leq 1$, then the sequence $\left\{x_{k}\right\}_{k \in N}$ generated by the predictor-corrector algorithm is bounded and $\lim _{k \rightarrow \infty}\left\|x^{k}-x^{k+1}\right\|=0$.

Proposition 23. Assume that $\alpha_{k} \geq \alpha>0$ for all $k \in N$. If the sequence $\left\{x_{k}\right\}_{k \in N}$ generated by the predictor-corrector algorithm is bounded and $\lim _{k \rightarrow \infty}\left\|x^{k}-x^{k+1}\right\|=0$, then every limit point of $\left\{x^{k}\right\}_{k \in N}$ is a solution of (VIP).

Second case is under imperfect foresight.

Assumption 24. Assume that there exist $r, c, d>0$ and $r \geq$ $\max \{d, c / \mu\}$, for all $x, y, z \in C$ :

(i) $\langle F(x), y-x\rangle \geq 0 \Rightarrow\langle F(y), x-y\rangle \leq-r\|x-y\|^{2}$;

(ii) $\langle F(y)-F(z), x-y\rangle \leq c\|y-z\|^{2}+d\|x-y\|^{2}$.

We denote

$$
\begin{aligned}
& A_{k}= \frac{\alpha_{k} d}{\mu}+\frac{\mu}{2} ; \\
& B_{k}= 2 \mu \alpha_{k} c+2 \alpha_{k} d+L \mu ; \\
& C_{k}= \frac{\alpha_{k} d}{\mu}+\frac{\mu}{2}+\frac{d-r}{4}+\frac{c}{2 \mu} ; \\
& D_{k}= 2 \mu \alpha_{k} c+2 \alpha_{k} d+L \mu+4 c \alpha_{k} ; \\
& E_{k}=\min \left\{\frac{\beta-B_{k}}{\alpha_{k}}+\frac{r \mu-c}{2 \mu} ; \frac{\beta-C_{k}}{\alpha_{k}} ;\right. \\
&\left.\quad \frac{(1+8 \mu) \beta-D_{k}-8 \mu B_{k}}{8 \mu \alpha_{k}}\right\} .
\end{aligned}
$$

Theorem 25. Suppose that $F(x)$ satisfies Assumption 24 and $\beta \geq \max \left\{A_{k}, B_{k}, C_{k}, D_{k}\right\}, \varepsilon_{k} \leq E_{k}\left\|x^{k}-x^{k+}\right\|^{2}$; then the sequence $\left\{x^{k}\right\}_{k \in N}$ generated by the predictor-corrector methods is bounded and $\lim _{k \rightarrow \infty}\left\|x^{k}-x^{k+1}\right\|=0$.

Theorem 26. Assume that $\alpha_{k} \geq \alpha>0$ for all $k \in N$. If the sequence $\left\{x_{k}\right\}_{k \in N}$ generated by the predictor-corrector algorithm is bounded and $\lim _{k \rightarrow \infty}\left\|x^{k}-x^{k+1}\right\|=0$, then every limit point of $\left\{x^{k}\right\}_{k \in N}$ is a solution of (VIP). 
Similar to Theorems 15 and 16, we can prove Theorems 25 and 26 . Here, we will omit their details.

Moreover, we can also give a stopping criterion.

Definition 27. Let $\Delta \geq 0$. A point $x^{*}$ is called a $\Delta$-stationary point of problem (VIP) if $x^{*} \in C$ and

$$
\exists \gamma \in \partial_{\Delta}\left(\left\langle F\left(x^{*}\right), \cdot-x^{*}\right\rangle+\varphi_{C}\right)\left(x^{*}\right) \text {, s.t. }\|\gamma\| \leq \Delta .
$$

Proposition 28 (see [20]). Let $x^{k+}=\arg \min _{y \in C}\left\{\alpha_{k} \bar{f}\left(x^{k}\right.\right.$, $\left.y)+h(y)-h\left(x^{k}\right)-\left\langle\nabla h\left(x^{k}\right), y-x^{k}\right\rangle\right\} ; \gamma^{k}=\left(1 / \alpha_{k}\right)\left[\nabla h\left(x^{k}\right)-\right.$ $\left.\nabla h\left(x^{k+}\right)\right]=\left(1 / \alpha_{k}\right)\left[\nabla h\left(x^{k}\right)-\nabla h\left(x^{k+1}\right)\right] ; \delta^{k}=\left\langle\gamma_{k}^{i}, x^{k+}-x^{k}\right\rangle-$ $\bar{f}\left(x^{k}, x^{k+}\right)$.

Then $\delta^{k} \geq 0$ and $\gamma^{k} \in \partial_{\delta^{k}}\left(\left\langle F\left(x^{k}\right), \cdot-x^{k}\right\rangle+\psi_{c}\right)\left(x^{k}\right)$.

Theorem 29. Assume that $\alpha_{k} \geq \alpha>0$ for all $k \in N$ and that the assumptions of Theorem 25 hold. Let $\left\{x^{k}\right\}_{k \in N}$ be generated by the predictor-corrector algorithm, then the sequences $\left\{\gamma^{k}\right\}_{k \in N}$ and $\left\{\delta^{k}\right\}_{k \in N}$ converge to zero.

Likewise, we omit the proof.

Finally, we have the predictor-corrector algorithm for variational inequalities problems as follows.

Algorithm 30 (the predictor-corrector algorithms for (VIP)). Let $\alpha_{k} \geq \alpha>0, \varepsilon_{k}>0$, for all $k \in N$.

Step 1. Let $k=0, x^{0} \in C$, and $\delta>0$.

Step 2. Find a $\mu$-approximation $\bar{f}(x, \cdot)$ of $f(x, \cdot)=\langle F(x), \cdot-$ $x\rangle$ at $x$ by predictor-corrector method. Let

$$
\begin{aligned}
& x^{k+}=\min _{y \in C}\left\{\alpha_{k} \bar{f}\left(x^{k}, y\right)+H\left(x^{k}, y\right)\right\} ; \\
& \varepsilon_{k} \leq E_{K}\left\|x^{k}-x^{k+}\right\|^{2} ; \\
& x^{k+1} \in \varepsilon_{k}-\min _{y \in C}\left\{\alpha_{k} f\left(x^{k+}, y\right)+H\left(x^{k+}, y\right)\right\} .
\end{aligned}
$$

Step 3. If $\left\|x^{k}-x^{k+1}\right\|^{2}<\delta$, then stop; otherwise put $k=k+1$ and go to Step 2 .

\section{Conclusions}

In this paper, we mainly present a predictor-corrector method for solving nonsmooth convex equilibrium problems based on the auxiliary problem principle. In the main algorithm each stage of computation requires two proximal steps. One step serves to predict the next point; the other helps to correct the new prediction. This method can operate well in practice. At the same time, we present convergence analysis under perfect foresight and imperfect one. In particular, we introduce a stopping criterion which gives rise to $\Delta$ stationary points. Moreover, we apply this algorithm for solving the particular case: variational inequalities.

For further work, the need can be anticipated: here we only give the conceptual algorithmic framework to solve this class of (EP), we will continue to study its rapidly convergent executable algorithm, and we will consider how to use bundle techniques to approximate proximal points and other related quantities. Moreover, we will strive to extend the nonsmooth convex equilibrium problems to nonconvex cases, where its related theory will be researched in later papers.

\section{Conflict of Interests}

The authors declare that there is no conflict of interests regarding the publication of this paper.

\section{References}

[1] E. Blum and W. Oettli, "From optimization and variational inequalities to equilibrium problems," The Mathematics Student, vol. 63, no. 1-4, pp. 123-145, 1994.

[2] F. Giannessi and A. Maugeri, Variational Inequalities and Network Equilibrium Problems, Plenum Press, New York, NY, USA, 1995.

[3] F. Giannessi, A. Maugeri, and P. M. Pardalos, Equilibrium Problems: Nonsmooth Optimization and Variational Inequality Models, Kluwer Academic, Dordrecht, The Netherlands, 2001.

[4] M. A. Noor and W. Oettli, "On general nonlinear complementarity problems and quasi-equilibria," Le Matematiche, vol. 49, no. 2, pp. 313-331 (1995), 1994.

[5] J. Eckstein, "Nonlinear proximal point algorithms using Bregman functions, with applications to convex programming," Mathematics of Operations Research, vol. 18, no. 1, pp. 202-226, 1993.

[6] R. Glowinski, J. L. Lions, and R. Tremolieres, Numerical Analysis of Variational Inequalities, North-Holland, Amsterdam, The Netherlands, 1981.

[7] D. Kinderlehrer and G. Stampacchia, An Introduction to Variational Inequalities and Their Applications, Academic Press, London, UK, 1980.

[8] M. A. Noor, "Some recent advances in variational inequalitiespart 1: basic concepts," New Zealand Journal of Mathematics, vol. 26, no. 1, pp. 53-80, 1997.

[9] M. A. Noor, "Some recent advances in variational inequalities. Part 2: other concepts," New Zealand Journal of Mathematics, vol. 26, pp. 229-255, 1997.

[10] M. Patriksson, Nonlinear Programming and Variational Inequality Problems: A Unified Approach, Kluwer Academic, Dordrecht, The Netherlands, 1999.

[11] R. T. Rockafellar, "Monotone operators and the proximal point algorithm," SIAM Journal on Control and Optimization, vol. 14, no. 5, pp. 877-898, 1976.

[12] D. L. Zhu and P. Marcotte, "Co-coercivity and its role in the convergence of iterative schemes for solving variational inequalities," SIAM Journal on Optimization, vol. 6, no. 3, pp. 714-726, 1996.

[13] A. N. Iusem and W. Sosa, "Iterative algorithms for equilibrium problems," Optimization, vol. 52, no. 3, pp. 301-316, 2003.

[14] H. Brezis, L. Nirenberg, and G. Stampacchia, "A remark on Ky Fan's minimax principle," Bollettino della Unione Matematica Italiana, vol. 6, pp. 293-300, 1972.

[15] A. N. Iusem and W. Sosa, "New existence results for equilibrium problems," Nonlinear Analysis. Theory: Methods \& Applications., vol. 52, no. 2, pp. 621-635, 2003.

[16] M. A. Noor, "Extragradient methods for pseudomonotone variational inequalities," Journal of Optimization Theory and Applications, vol. 117, no. 3, pp. 475-488, 2003. 
[17] M. A. Noor, M. Akhter, and K. I. Noor, "Inertial proximal method for mixed quasi variational inequalities," Nonlinear Functional Analysis and Applications, vol. 8, no. 3, pp. 489-496, 2003.

[18] N. El Farouq, "Pseudomonotone variational inequalities: convergence of proximal methods," Journal of Optimization Theory and Applications, vol. 109, no. 2, pp. 311-326, 2001.

[19] S. D. Flåm and A. S. Antipin, "Equilibrium programming using proximal-like algorithms," Mathematical Programming, vol. 78, no. 1, pp. 29-41, 1997.

[20] T. T. V. Nguyen, J. J. Strodiot, and V. H. Nguyen, "A bundle method for solving equilibrium problems," Mathematical Programming B, vol. 116, no. 1-2, pp. 529-552, 2009.

[21] G. Stampacchia, "Formes bilineaires coercitives sur les ensembles convexes," Comptes Rendus de l'Académie des Sciences, vol. 258, pp. 4413-4416, 1964.

[22] M. Bounkhel, L. Tadj, and A. Hamdi, "Iterative schemes to solve nonconvex variational problems," Journal of Inequalities in Pure and Applied Mathematics, vol. 4, pp. 1-14, 2003.

[23] F. H. Clarke, Y. S. Ledyaev, and P. R. Wolenski, Nonsmooth Analysis and Control Theory, Springer, Berlin, Germany, 1998.

[24] D. Kinderlehrer and G. Stampacchia, An Introduction to Variational Inequalities and Their Applications, SIAM, Philadelphia, $\mathrm{Pa}$, USA, 2000.

[25] M. A. Noor, "General variational inequalities," Applied Mathematics Letters, vol. 1, no. 2, pp. 119-122, 1988.

[26] M. A. Noor, "Some developments in general variational inequalities," Applied Mathematics and Computation, vol. 152, no. 1, pp. 199-277, 2004.

[27] M. A. Noor, Principles of Variational Inequalities, Lap-Lambert Academic, Saarbrucken, Germany, 2009. 


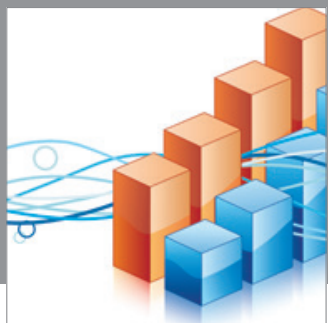

Advances in

Operations Research

mansans

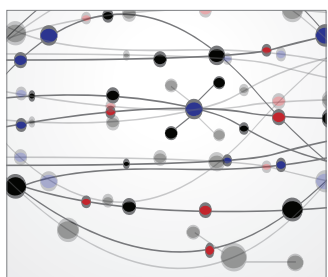

The Scientific World Journal
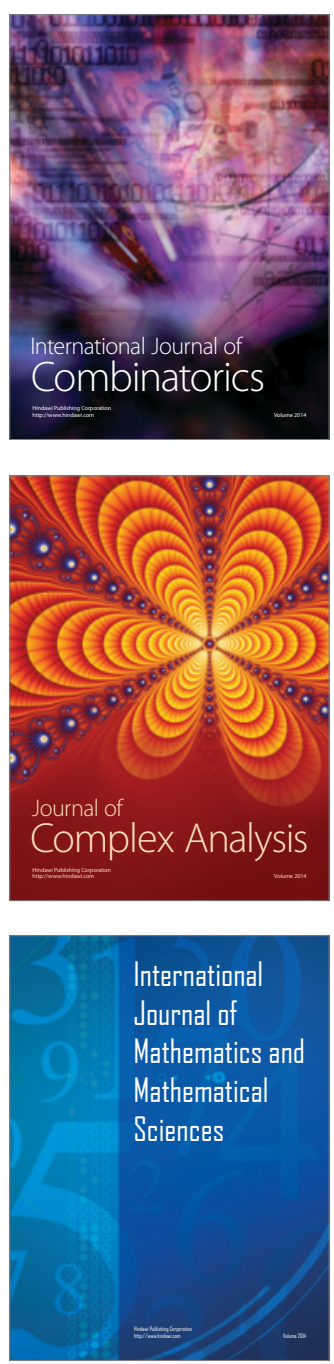
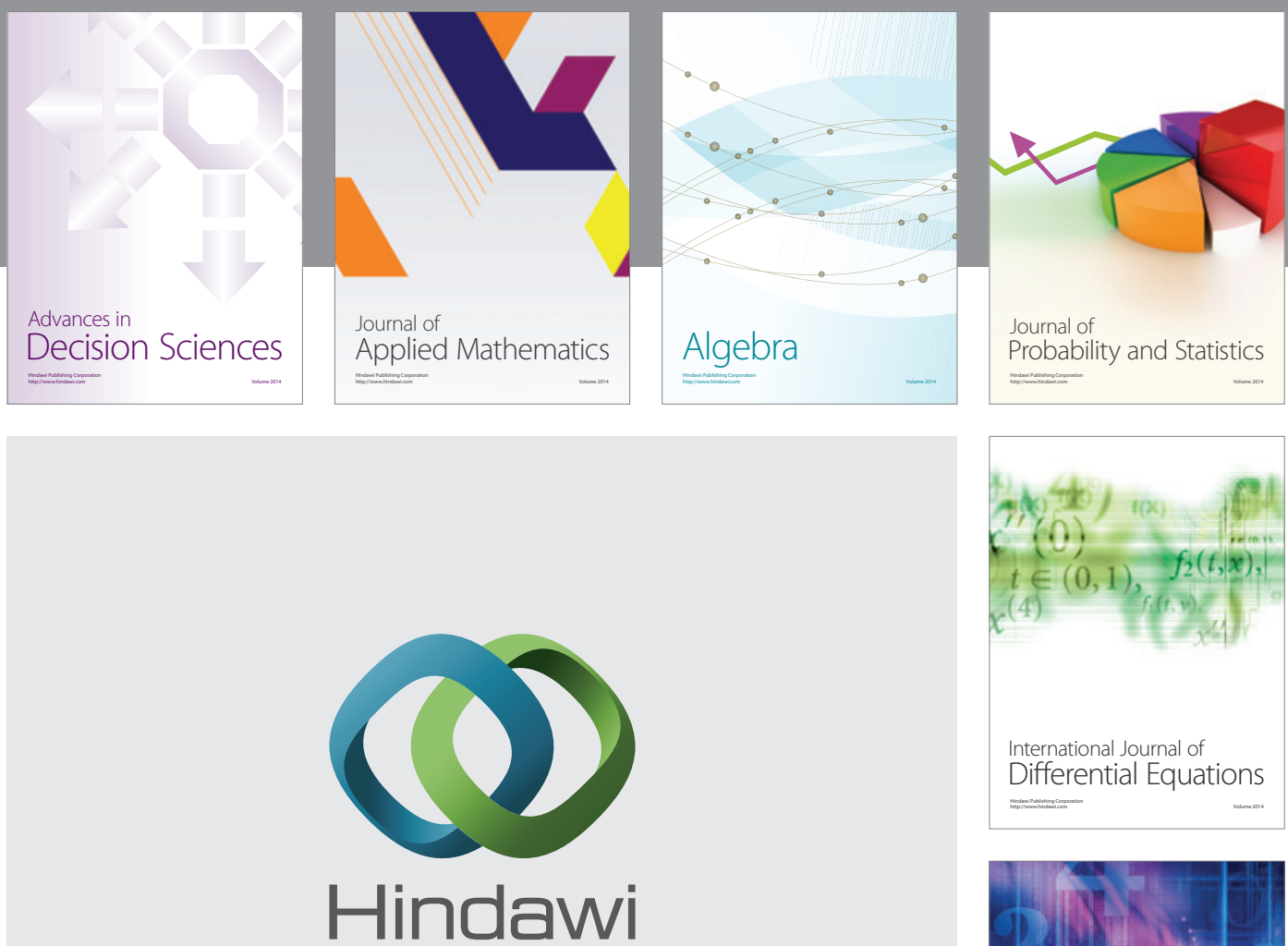

Submit your manuscripts at http://www.hindawi.com
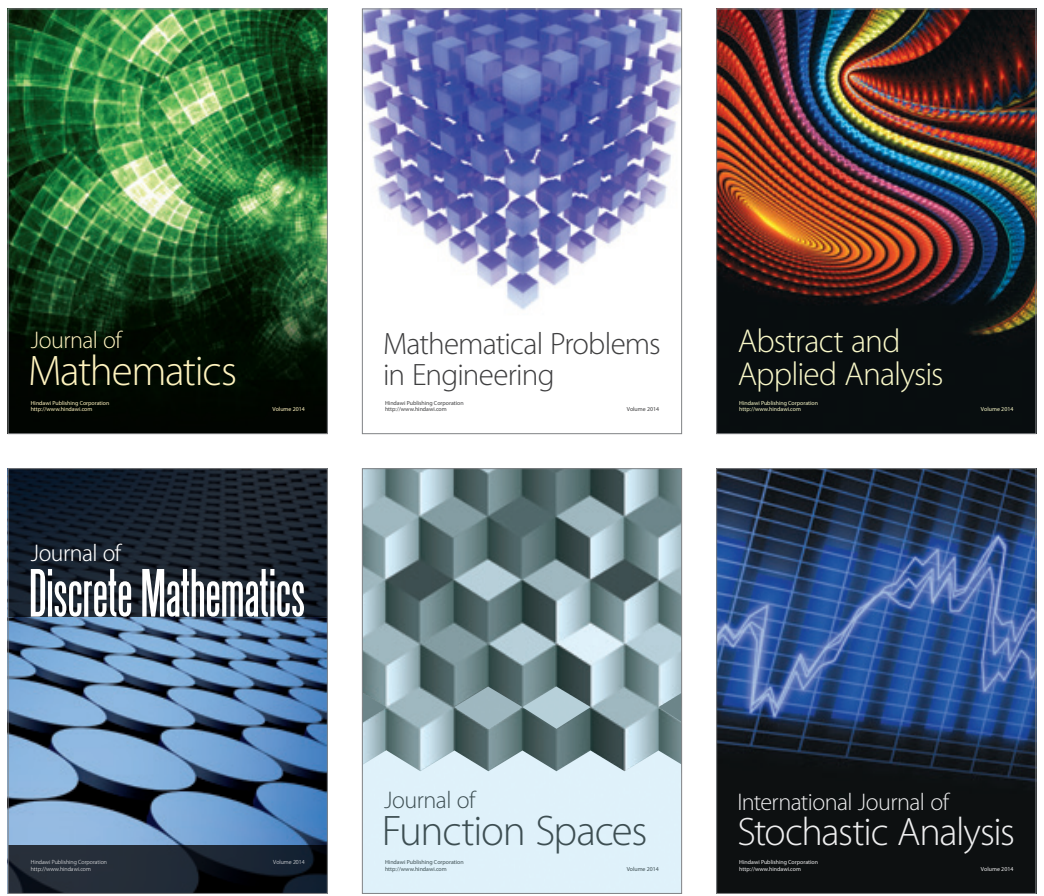

Journal of

Function Spaces

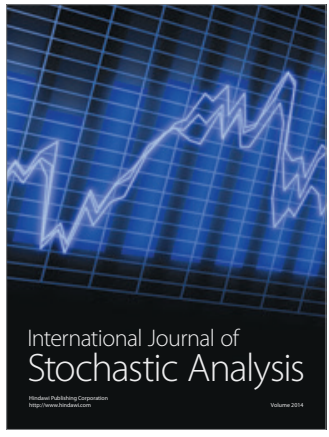

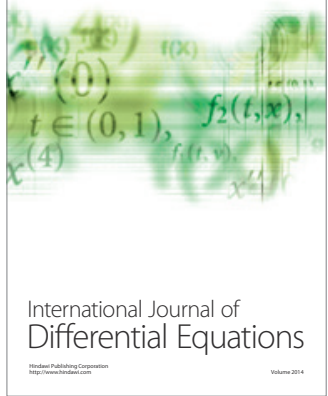
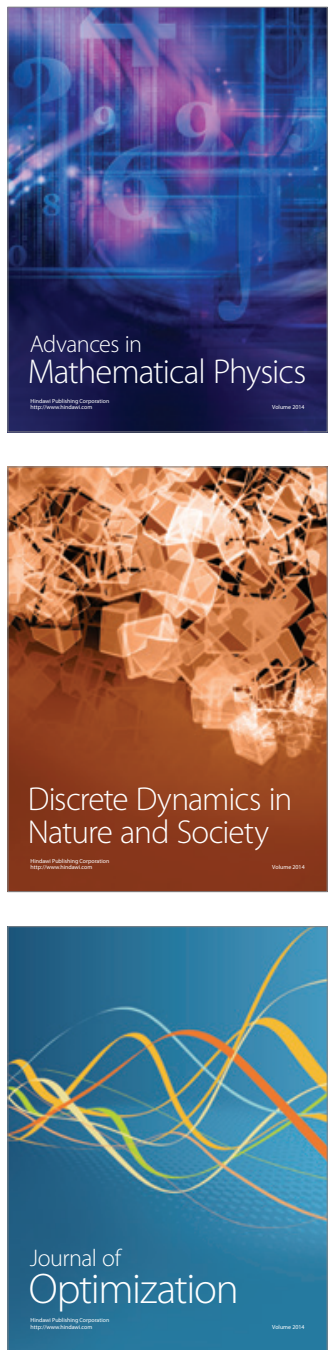